\title{
Performance Comparison of SISO and MIMO Low Level Controllers in a Special Trajectory Tracking Application*
}

\author{
Peter Bauer ${ }^{1}$ and Jozsef Bokor ${ }^{1}$
}

\begin{abstract}
This paper deals with the problem of low level aircraft controller design for monocular vision based sense and avoid task. The generated trajectory should be tracked through the yaw rate meanwhile about zero roll angle should be held to provide acceptable effective field of view to the camera. SISO PID and MIMO LQ controllers are designed for the Aerosonde UAV to track yaw rate and roll angle. Their performance is compared in linear and nonlinear simulations without and with trajectory tracking. Finally, the MIMO LQ method proves to be more suitable for the task.
\end{abstract}

\section{INTRODUCTION}

In monocular vision based sense and avoid problems for unmanned aerial vehicles (UAVs) the own aircraft carries a monocular camera with which the observation of the close other aerial vehicle (intruder) can be performed. After the detection of the intruder the estimation of its distance and flight direction should be done. This needs persistent excitation of the estimator (see [1] for example). Several other sources point out that lateral acceleration of the observer is required to provide intruder observability ([2], [3], [4]).

In a previous work [1] a zig-zag trajectory defined by its corner points was used to give persistent excitation to the estimator (see Fig. 7). However, the tracking of this trajectory can cause to loose the intruder from camera field of view (FOV) because of rotation and translation of the aircraft.

Another work ([5]) points out that tracking of a zigzag trajectory with an unconventional tracker can solve the problem. This tracker should be designed to implement the following functionalities:

1) Track the trajectory by generating a yaw rate reference from the course angle difference $\left(r_{r e f}=K_{r} \cdot\left(\chi_{\text {ref }}-\right.\right.$ $\chi)$ where $\chi_{\text {ref }}$ reference, $\chi$ actual course angle and $K_{r}$ gain) and following it.

2) Attempt to hold zero roll angle $(\phi)$. This will probably result in large angle of sideslip $(\beta)$.

This can be called unconventional because uses rudder and large sideslip angles to track the trajectory instead rolling the aircraft with aileron. In [6] a hardware-in-the-loop (HIL) demonstration setup is built which simulates aircraft motion together with image generation and processing, intruder state estimation and collision probability calculation. In this HIL

IEEE ID of final version: 978-1-4799-5899-3/14/\$31.00 (C)2014 IEEE, published in proceedings of IEEE MED'14 pp. 1293-1298

*This work is supported by the Office of Naval Research Global, Grant Number N62909-10-1-7081, Dr. Paul Losiewicz program officer.

${ }^{1}$ Author is with Institute for Computer Science and Control, Hungarian Academy of Sciences, Budapest, Hungary peter.baueresztaki.mta.hu

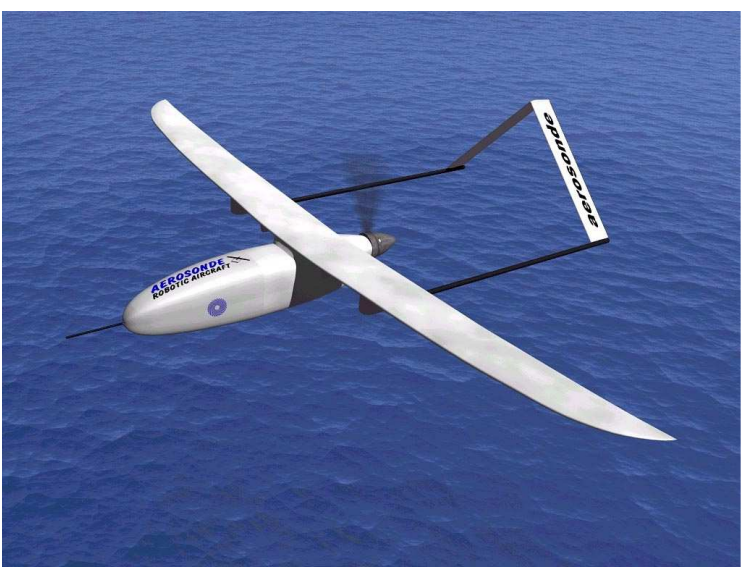

Fig. 1. Photo of the Aerosonde UAV

setup the model of the Aerosonde UAV from [7] is used. However, this UAV shows special dynamic characteristics which makes it challenging to implement the low level controllers of the above described tracking method with multi loop SISO PID control solutions. That's why the model should be examined in detail and possibly MIMO controller design should be applied to achieve acceptable performance. This is the topic of this paper.

The paper starts with the introduction of the aircraft model in section II then it examines the possible implementation of the unconventional tracker with SISO PID low level controllers in section III. In the next section (IV) it deals with the implementation applying a MIMO LQ optimal tracker solution in the low level control. Section V compares the tracking results with the two different low level control solutions. Finally section VI concludes the paper.

\section{LATERAL-DIRECTIONAL DYNAMICS OF THE AEROSONDE UAV}

The photograph of Aerosonde UAV can be seen in Fig. 1 . The figure shows that it is equipped with inverted V-tail and ruddervators so its dynamics can be special.

The parameters of its linearized aerodynamic model together with mass, inertia and geometric data are given in [7] Appendix D. Here, only the nonzero parameters related to the lateral-directional dynamics of the aircraft $(\mathrm{A} / \mathrm{C})$ are repeated in table I using standard notations.

Only the lateral-directional dynamics is considered and controlled in this paper, the longitudinal controllers are 
TABLE I

PARAMETERS OF AEROSONDE UAV

\begin{tabular}{|c|c|c|c|c|c|}
\hline Param. & Value & Param. & Value & Param. & Value \\
\hline $\mathrm{m}$ & $1.56 \mathrm{~kg}$ & $C_{Y_{\beta}}$ & -0.98 & $C_{l_{\delta_{r}}}$ & 0.105 \\
\hline$J_{x}$ & $0.1147 \mathrm{kgm}^{2}$ & $C_{Y_{\delta_{r}}}$ & -0.17 & $C_{n_{\beta}}$ & 0.25 \\
\hline$J_{z}$ & $0.1712 \mathrm{kgm}^{2}$ & $C_{l_{\beta}}$ & -0.12 & $C_{n_{p}}$ & 0.022 \\
\hline$J_{x z}$ & $0.0015 \mathrm{kgm}^{2}$ & $C_{l_{p}}$ & -0.26 & $C_{n_{r}}$ & -0.35 \\
\hline $\mathrm{S}$ & $0.2589 \mathrm{~m}^{2}$ & $C_{l_{r}}$ & 0.14 & $C_{n_{\delta_{a}}}$ & 0.06 \\
\hline $\mathrm{b}$ & $1.4224 \mathrm{~m}$ & $C_{l_{\delta_{a}}}$ & 0.08 & $C_{n_{\delta_{r}}}$ & -0.032 \\
\hline
\end{tabular}

implemented unchanged from [7] (indicated airspeed and altitude hold). In the final tests in HIL simulation all of the controllers are applied together.

Table I shows that the aircraft has special dynamics because the aileron $\left(\delta_{a}\right)$ has larger effect on yaw than the rudder $\left(\delta_{r}\right)\left(C_{n_{\delta_{a}}}\right.$ is larger than $\left.\left|C_{n_{\delta_{r}}}\right|\right)$ and the rudder has larger effect on roll than the aileron $\left(C_{l_{\delta_{r}}}\right.$ is larger than $\left.C_{l_{\delta_{a}}}\right)$.

The linear state space lateral-directional model of Aerosonde can be built based on [7] chapter 5.5 considering the conversion between the derivative of lateral velocity $(v)$ and angle of sideslip:

$$
\dot{\beta} \approx 1 / V_{a} \cdot \dot{v}
$$

Here $V_{a}$ is the air relative velocity of $\mathrm{A} / \mathrm{C}$. In the linear model it was set to $22 \mathrm{~m} / \mathrm{s}$ which is the reference $V_{a}$ value in the HIL simulation tracked by the longitudinal controller. Considering the coefficients in table I the state vector of the model was selected to be $x=\left[\begin{array}{llll}\beta & p & r & \phi\end{array}\right]^{T}$ where $p$ and $r$ are the roll and yaw rates respectively. The roll angle will be a tracking variable that's why it is added to the model simply considering it to be the integral of roll rate. The input vector can be defined as $u=\left[\begin{array}{ll}\delta_{a} & \delta_{r}\end{array}\right]^{T}$ and full state measurement can be assumed.

The continuous time linear state space model was transformed into discrete time applying a $T_{s}=0.01 \mathrm{~s}$ sampling time (100 Hz sampling). The matrices of the discrete time model are as follows:

$$
\begin{aligned}
& {\left[\begin{array}{l}
\beta \\
p \\
r \\
\phi
\end{array}\right]_{k+1}=A\left[\begin{array}{l}
\beta \\
p \\
r \\
\phi
\end{array}\right]_{k}+B\left[\begin{array}{l}
\delta_{a} \\
\delta_{r}
\end{array}\right]_{k}} \\
& A=\left[\begin{array}{cccc}
0.9913 & 0 & -0.0097 & 0 \\
-0.5694 & 0.9031 & 0.0450 & 0 \\
0.6314 & -0.0027 & 0.9378 & 0 \\
-0.0029 & 0.0095 & 0.0002 & 1
\end{array}\right] \\
& B=\left[\begin{array}{cc}
-0.0010 & -0.0007 \\
0.4834 & 0.5846 \\
0.1943 & -0.0465 \\
0.0025 & 0.0030
\end{array}\right]
\end{aligned}
$$

This also shows that the effect of aileron on the yaw dynamics is larger than the effect of rudder $(B(3,1)>$ $|B(3,2)|)$ and the effect of rudder on roll dynamics is larger than the effect of aileron $(B(2,2)>B(2,1))$.
Checking the controllability of the system shows that it is controllable. The poles of the system are:

$$
p=\left[\begin{array}{llll}
1 & 0.9 & 0.965+0.074 i & 0.965-0.074 i
\end{array}\right]
$$

The 1 pole clearly shows the integrator from roll rate to roll angle. The other poles are stable.

Both the SISO PID and the MIMO LQ low level trackers are first tuned and tested on this linear state space model then they are applied in the trajectory tracking part of the sense and avoid HIL simulation where the nonlinear Aerosonde model is used (see [6]).

The next section deals with the tuning of the PID low level controllers while the section after deals with the LQ tracking controller.

\section{TRACKER IMPLEMENTATION WITH SISO PID LOW LEVEL CONTROLLERS}

The trajectory tracking lateral control of the Aerosende UAV can be implemented with cascaded control loops. The highest level manages the waypoints and calculates the reference course angle $\chi_{c}$ of the aircraft from $\mathrm{A} / \mathrm{C}$ and waypoint (WP) north (x) / east (y) position as follows:

$$
\chi_{c}=\arctan 2\left(\frac{y_{W P}-y_{A / C}}{x_{W P}-x_{A / C}}\right)
$$

The next level considers the difference between the reference and actual course angle and generates a yaw rate reference from it $\left(r_{r e f}=K_{r} \cdot\left(\chi_{\text {ref }}-\chi\right)\right)$. The low level lateral-directional controllers should follow this yaw rate reference and hold the roll angle of the aircraft around zero.

With SISO PID control an aileron and a rudder control channel should be implemented separately considering the yaw rate and roll angle tracking errors. The two errors can be connected in two different ways to the actuators and so two possible solutions exist:

1) C1: Track the roll angle with aileron and the yaw rate with rudder (instead of the usually tracked (zero) sideslip angle). Controlling the roll dynamics with aileron and the yaw dynamics with rudder is the usual solution in A/C lateral controller design.

2) C2: Track the roll angle with rudder and the yaw rate with aileron. In case of the Aerosonde UAV this could possibly provide better tracking results because of larger rudder effectiveness in the roll and aileron effectiveness in the yaw channel.

All two versions are tuned and compared in this work applying PI control in all channels. The discrete time implementation of PI control is as follows:

$$
\begin{aligned}
& \delta(i)_{k}=K_{P}(j) \cdot e(j)_{k}+K_{I}(j) \cdot I(j)_{k} \\
& I(j)_{k+1}=I(j)_{k}+A W \cdot T_{s} \cdot e(j)_{k}
\end{aligned}
$$

Here $i$ can be aileron or rudder and $j$ can be $r$ or $\phi$. $A W$ is an anti-windup constant which is 0 if $\mid K_{P}(j) \cdot e(j)_{k}+$ $K_{I}(j) \cdot I(j)_{k} \mid>\delta(i)_{L I M}$ and 1 otherwise. This means that 
integration is stopped if the actuator deflection command reached the limit.

For the Aerosonde UAV both the aileron and rudder deflection limits were considered as $\pm 45^{\circ}$.

The controller gains in the two cases were tuned by trial and error using the simulation of the linear state space model. The final gains are summarized in table II. The table shows that negative gains were required in yaw control by rudder because of negative rudder effectiveness.

The designed controllers were first tested on the linear system, then tested and re-tuned on the nonlinear. The comparison of controllers is presented in section V.

\section{TRACKER IMPLEMENTATION WITH MIMO LQ LOW LEVEL CONTROLLER}

In this section the implementation of the low level (yaw rate and zero roll angle) controller is done using a linear quadratic (LQ) sub-optimal tracker solution developed by the authors in [8]. The higher level controller is the same as for the PID solutions. The steps of the LQ controller design and the final expressions are briefly repeated here.

1) Design a stabilizing state feedback controller for the pair $(A, B)$ in (2) if required: $x_{k+1}=\underbrace{\left(A-B K_{x 1}\right)}_{\Phi} x_{k}+B u_{k}$

2) Determine the solution of the steady state constant reference tracking problem considering the stabilized system

$$
\begin{aligned}
& (I-\Phi) x_{\infty}=B u_{\infty} \rightarrow x_{\infty}=(I-\Phi)^{-1} B u_{\infty} \\
& C_{r} x_{\infty}=\underbrace{C_{r}(I-\Phi)^{-1} B}_{F} u_{\infty}=r_{\infty} \\
& u_{\infty}=F^{+} r_{\infty}
\end{aligned}
$$

Here $y^{r}=C_{r} x$ is the tracking output of the system which should track the references and ()$^{+}$denotes the Moore-Penrose pseudoinverse of a matrix.

3) Construct an LQ sub-optimal tracking controller for time-varying references, centering the original system with the steady state equilibrium point and the steady state reference value. This leads to the following functional for the centered system:

$$
\begin{aligned}
& J(\Delta x, \Delta \tilde{x}, \Delta u)= \\
& =\frac{1}{2} \sum_{k=0}^{\infty}\left(\left(\Delta x_{k}-\Delta \tilde{x}_{k}\right)^{T} Q\left(\Delta x_{k}-\Delta \tilde{x}_{k}\right)+\right. \\
& \left.+\Delta u_{k}^{T} R \Delta u_{k}\right) \\
& \text { where }: \quad Q=\bar{C}^{T} Q_{1} \bar{C}+C_{r}^{T} Q_{2} C_{r}
\end{aligned}
$$

Here $\Delta x_{k}=x_{k}-x_{\infty}, \Delta \tilde{x}_{k}=C_{r}^{T}\left(C_{r} C_{r}^{T}\right)^{-1}\left(r_{k}-r_{\infty}\right)$, $\Delta u_{k}=u_{k}-u_{\infty}$ and $\bar{C}=\left(I-C_{r}^{T}\left(C_{r} C_{r}^{T}\right)^{-1} C_{r}\right) \cdot Q_{1}$, $Q_{2}$ and $R$ are user defined weights. $Q_{2}$ weights the tracking error, $Q_{1}$ weights the states which do not affect $y^{r}$ and $R$ weights the control energy.
TABLE II

PID CONTROL PARAMETERS (LINEAR MODEL)

\begin{tabular}{|c|c|c|c|c|c|}
\hline & & \multicolumn{2}{|c|}{ Yaw control } & \multicolumn{2}{c|}{ Roll control } \\
\hline & Actuator & $K_{P}(r)$ & $K_{I}(r)$ & $K_{P}(\phi)$ & $K_{I}(\phi)$ \\
\hline \hline \multirow{2}{*}{ C1 } & $\delta_{r}$ & -2 & -4 & - & - \\
\cline { 2 - 6 } & $\delta_{a}$ & - & - & 0.5 & 2.5 \\
\hline \hline \multirow{2}{*}{ C2 } & $\delta_{r}$ & - & - & 1.5 & 3 \\
\cline { 2 - 6 } & $\delta_{a}$ & 1 & 2 & - & - \\
\hline
\end{tabular}

The final input to control the original system in (2) results by summarizing all of the inputs from step 1 to 3 (considering $r_{\infty}=r_{k+1}$ ):

$$
u_{k}=-K_{x} x_{k}-K_{S_{2}}\left(r_{k+1}-r_{k}\right)+K_{r_{\infty}} r_{k+1}
$$

In this application the tracking outputs are the $\phi$ roll angle and $r$ yaw rate so $C_{r}=\left[\begin{array}{llll}0 & 0 & 0 & 1 \\ 0 & 0 & 1 & 0\end{array}\right]$. The unaffected states are $p$ and $\beta$.

In the control design $Q_{1}=0$ was selected (no weights on unaffected states). The other weights were selected by applying Bryson's method. $\pm 6^{\circ}$ error was allowed for $\phi$ and $\pm 2^{\circ} / s$ error for $r$ tracking. This way $Q_{2}=<100816>$ where $<>$ denotes a diagonal matrix. The control weights were chosen considering $\pm 25^{\circ}$ maximum actuator deflections $\left(R=<\begin{array}{ll}5.25 & 5.25\end{array}>\right.$ ) however, the saturation limits for aileron and rudder were again selected to be $\pm 45^{\circ}$.

The initial stabilizing controller is required ( $A$ has a pole on the unit circle). It was designed by pole placement prescribing the poles:

$$
\bar{p}=\left[\begin{array}{llll}
0.95 & 0.9 & 0.85 & 0.85
\end{array}\right]
$$

This means that the 1 pole was decreased to 0.95 , the 0.96 real parts were decreased to 0.85 (imaginary parts were removed) and 0.9 was left.

After tuning on the linear model finally $R=<5005>$ was selected ( $Q_{2}$ was unchanged). The designed controller was first tested on the linear system, then tested and retuned on the nonlinear one. The comparison with the other controllers is presented in the next section.

\section{COMPARISON OF THE DESIGNED CONTROLLERS}

In this section the two PID and the LQ low level controllers are compared first applying them on the linear lateraldirectional state space model. A doublet yaw rate reference is applied to test tracking quality. The second step was to test all controllers on the full nonlinear simulation of the Aerosonde UAV including longitudinal control and trajectory tracking. Finally, the effective camera field of view (EFOV) is defined and compared for the three solutions.

\section{A. Comparison of low level controllers applied on linear model}

The tracking results for yaw rate and roll angle are plotted in Fig.-s 2 and 3. From now on, the C1 PID controller is denoted by $P I D \delta_{a}$ and $\mathbf{C 2}$ by $P I D \delta_{r}$ in the figures. 


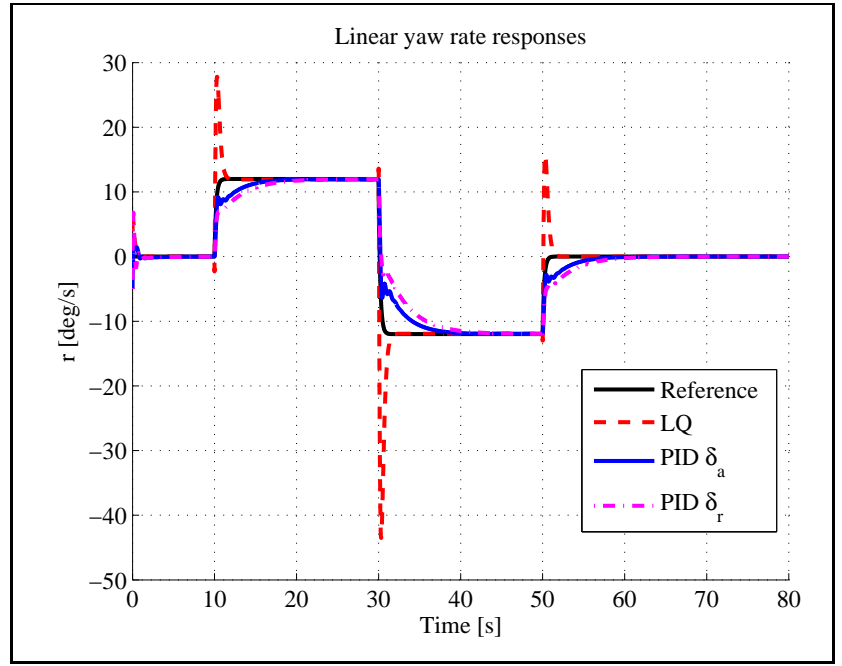

Fig. 2. Tracking of yaw rate reference in linear simulation

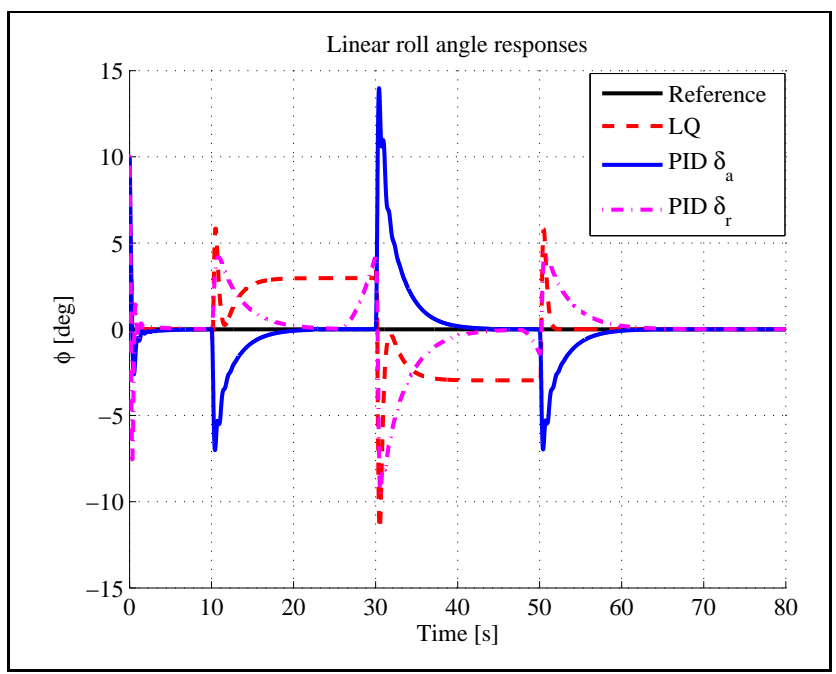

Fig. 3. Tracking of roll angle reference in linear simulation

Fig. 2 shows that the yaw rate is tracked by the two PID controllers equally well with asymptotic transient, meanwhile the LQ control has large overshoots at sudden reference changes but it is asymptotic after. This overshoot is partly caused by the dynamics of counteracting control surfaces to compensate roll motion. However, this can be useful in trajectory tracking when the course of the aircraft should be changed suddenly.

Fig. 3 shows that the dynamics of roll angle are similar with $L Q$ and $P I D \delta_{r}$ controllers. This shows that the MIMO controller automatically generates the solution which better fits the aircraft characteristics (larger rudder effectiveness in roll channel).

Considering all two figures the best solution is $P I D \delta_{r}$ which well tracks the yaw rate and produces the smallest roll angles.

In the next subsection the trajectory tracking results will be compared with all three controllers, from which the final best solution can be selected.

\section{B. Comparison of low level controllers applied in nonlinear model trajectory tracking}

In the tracking application all the controllers were re-tuned to hold the roll angle between $\pm 10^{\circ}$ and the yaw angle between $\pm 80^{\circ}$ which can give acceptable effective field of view. The final PID gains are summarized in table III.

TABLE III

PID CONTROL PARAMETERS (NONLINEAR MODEL)

\begin{tabular}{|c|c|c|c|c|c|}
\hline & & \multicolumn{2}{|c|}{ Yaw control } & \multicolumn{2}{c|}{ Roll control } \\
\hline & Actuator & $K_{P}(r)$ & $K_{I}(r)$ & $K_{P}(\phi)$ & $K_{I}(\phi)$ \\
\hline \hline \multirow{2}{*}{ C1 } & $\delta_{r}$ & -1 & -4 & - & - \\
\cline { 2 - 6 } & $\delta_{a}$ & - & - & 0.8 & 2.5 \\
\hline \hline \multirow{2}{*}{ C2 } & $\delta_{r}$ & - & - & 1.5 & 3 \\
\cline { 2 - 6 } & $\delta_{a}$ & 1.5 & 5 & - & - \\
\hline
\end{tabular}

In the LQ control the $R$ weight was changed to $R=<$ $10001000>$ to make the controller less aggressive. The $K_{r}$ tracking gain was 2 in every case. The reference yaw rate was saturated to $\pm 13^{\circ} / \mathrm{s}$ for $P I D \delta_{a}, \pm 15.5^{\circ} / \mathrm{s}$ for $P I D \delta_{r}$ and $\pm 17^{\circ} / s$ for $L Q$. The LQ tracker tolerated the largest saturation limit without oscillations.

The tracking results are plotted in Fig.-s 4 to 7.

Fig. 4 shows that the yaw rate overshoot is again present with the LQ method and the results with PID $\delta_{a}$ and $P I D \delta_{r}$ are now different.

In Fig. 5 the roll angles are between $\pm 10^{\circ}$ with every method, the dynamics of the controllers are different.

Fig. 6 shows that the yaw angle range is the smallest with the LQ controller so this is the best solution from this point of view.

Fig. 7 shows the tracking of the zig-zag trajectory with LQ and PID $\delta_{r}$ methods. The east coordinates of given waypoints are either $-50 \mathrm{~m}$ or $50 \mathrm{~m}$ the north coordinates are increased from $-1600 \mathrm{~m}$ in $200 \mathrm{~m}$ steps. The PID $\delta_{a}$ is not plotted because for that controller the forward step was increased to $230 \mathrm{~m}$ to limit the maximum yaw angles. This is because of the smallest maximum yaw rates with this method

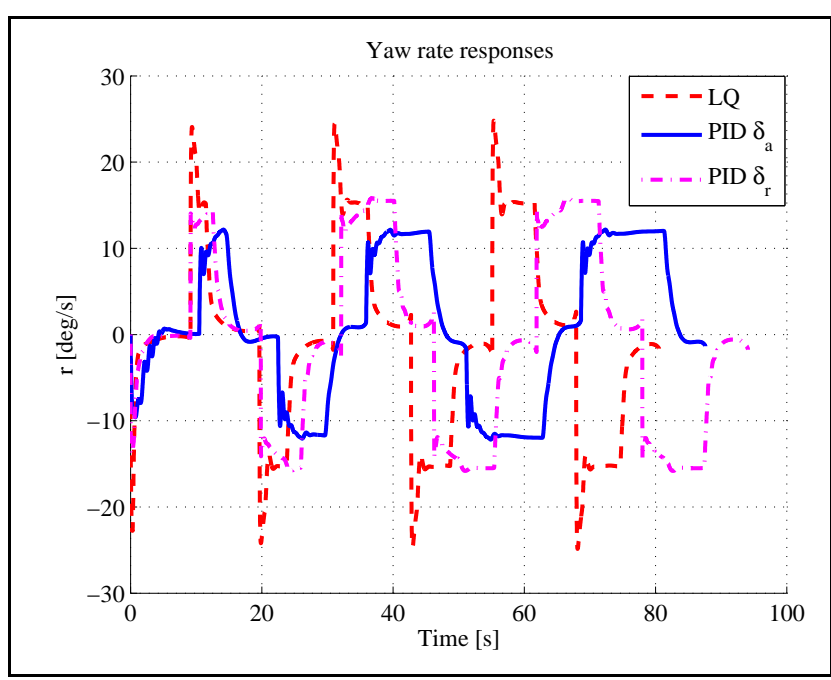

Fig. 4. Tracking of yaw rate reference during trajectory tracking 


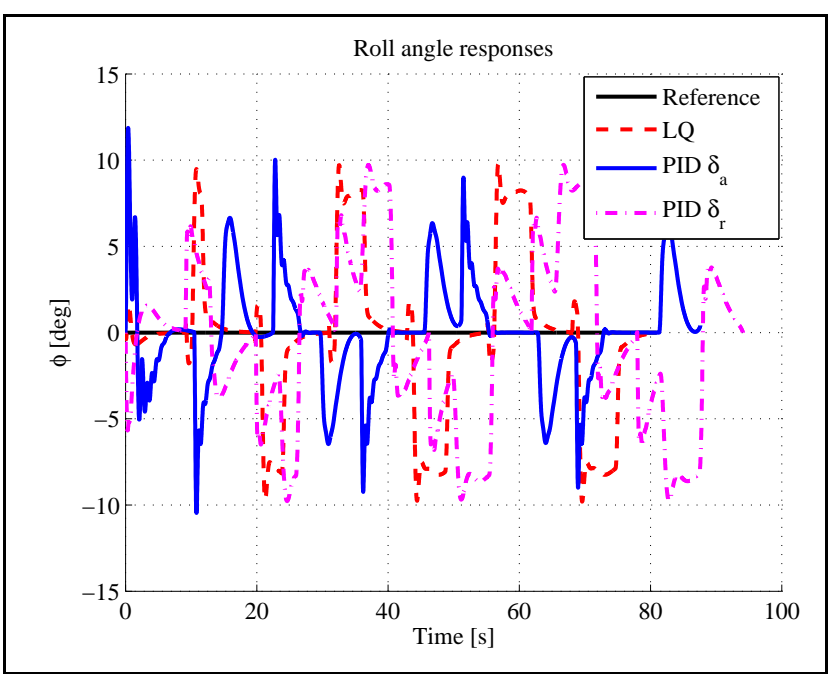

Fig. 5. Tracking of roll angle reference during trajectory tracking

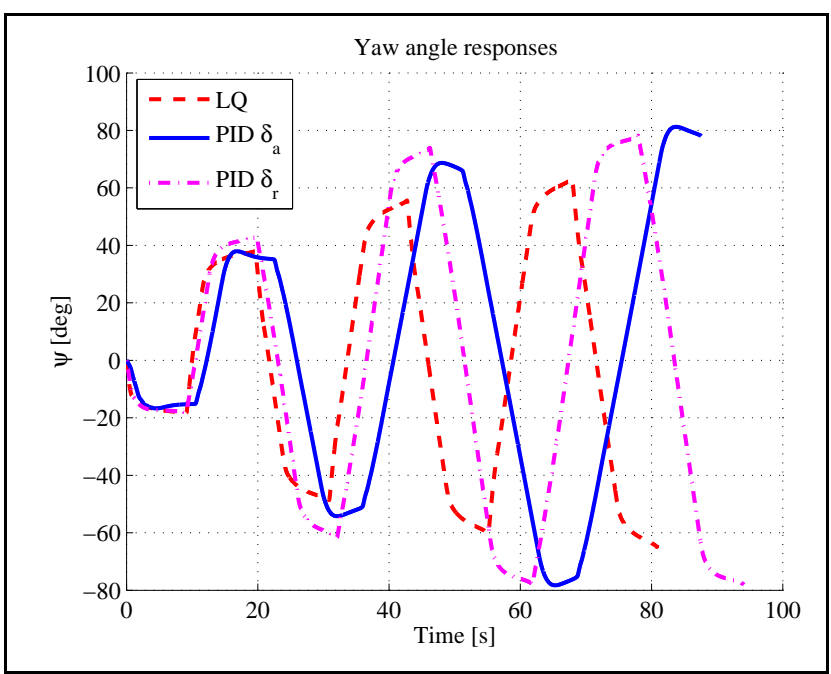

Fig. 6. Yaw angle during trajectory tracking

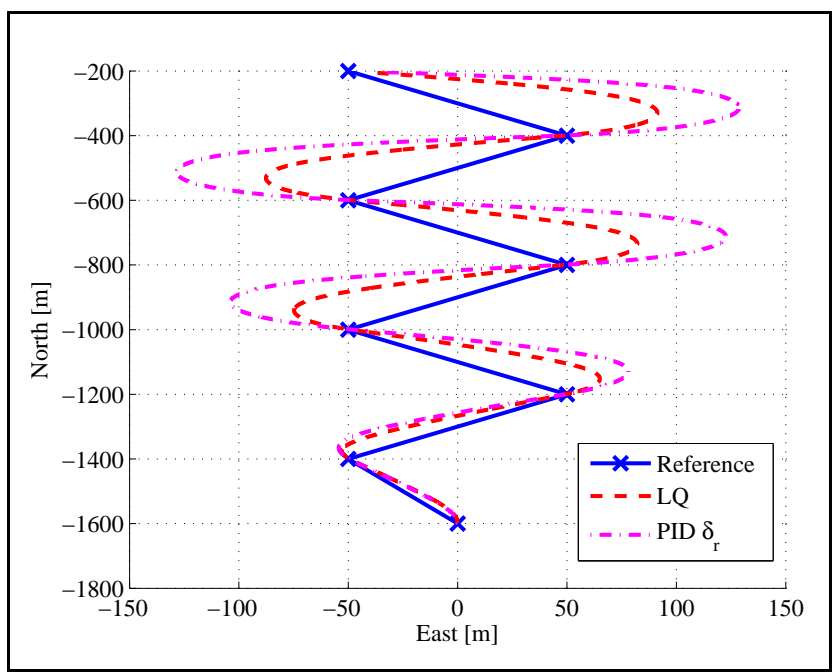

Fig. 7. Tracking of ZZ trajectory (see Fig. 4), however this results in worse excitation of the sense and avoid filter.

So, the final control solution can be selected from LQ and $P I D \delta_{r}$. The smaller yaw angles promise to have the MIMO LQ as the best solution but the final decision should be done based on the EFOVs.

\section{Comparison of effective field of views}

At first, define effective field of view (EFOV). Consider an aircraft with a fixed monocular camera (with a given field of view FOV). If one examines straight and level flight the camera views the same $3 \mathrm{D}$ region around the $\mathrm{A} / \mathrm{C}$ at all time (neglecting translation). However, if the aircraft rotates and translates the camera will view different $3 \mathrm{D}$ regions the union of which gives the EFOV. So EFOV is the region in front of the $\mathrm{A} / \mathrm{C}$ which is always seen by the camera irrespective of $\mathrm{A} / \mathrm{C}$ rotation or side and up/down translation.

In this work, only the change of EFOV with camera rotation is examined to compare the controllers. Camera FOV is represented on a spherical surface. EFOV was examined by determining the convex hull of the $\phi, \theta, \psi$ point set along an $\mathrm{A} / \mathrm{C}$ trajectory and calculating DCM-s in these points. The spherical surface with camera FOV was rotated with these DCMs (this means transformation into NorthEast-Down coordinate frame). Finally all the rotated FOVs were projected into the East-Down plane using Mercator projection (see [9]). In the resulting figures the intersection of the rotated regions shows the EFOV. The original camera FOV was $\pm 110^{\circ}$ horizontal and $\pm 30^{\circ}$ vertical (also plotted in the Fig.-s 8 to 10).

The figures show that the vertical range of EFOVs is very similar (the roll and pitch angles are similar) but the EFOV with LQ method is more wider. The pentagon like EFOVs can be approximated by rectangles (this is a bit conservative). The horizontal and vertical angular limits of these rectangular EFOVs are summarized in table IV.

TABLE IV

ZZ TRAJECTORY EFOVS

\begin{tabular}{|c||c|c|c|c|}
\hline Method & $-\beta$ & $\beta$ & $-\alpha$ & $\alpha$ \\
\hline$P I D \delta_{a}$ & -26.2 & 29.4 & -16.6 & 27.9 \\
\hline$P I D \delta_{r}$ & -29.7 & 30.1 & -18.5 & 22 \\
\hline LQ & -44.7 & 42.8 & -18.2 & 21.6 \\
\hline
\end{tabular}

Considering the EFOV results the LQ method has the best performance compared to the PID $\delta_{r}$ method, so the application of MIMO control design really improved performance.

\section{CONCLUSION}

This paper considers the problem of low level controller design for trajectory tracking for a UAV with special dynamic characteristics. The considered Aerosonde UAV has larger rudder effectiveness in the roll and larger aileron effectiveness in the yaw channel.

The trajectory and tracking requirements come from monocular vision based sense and avoid task of the UAV. 


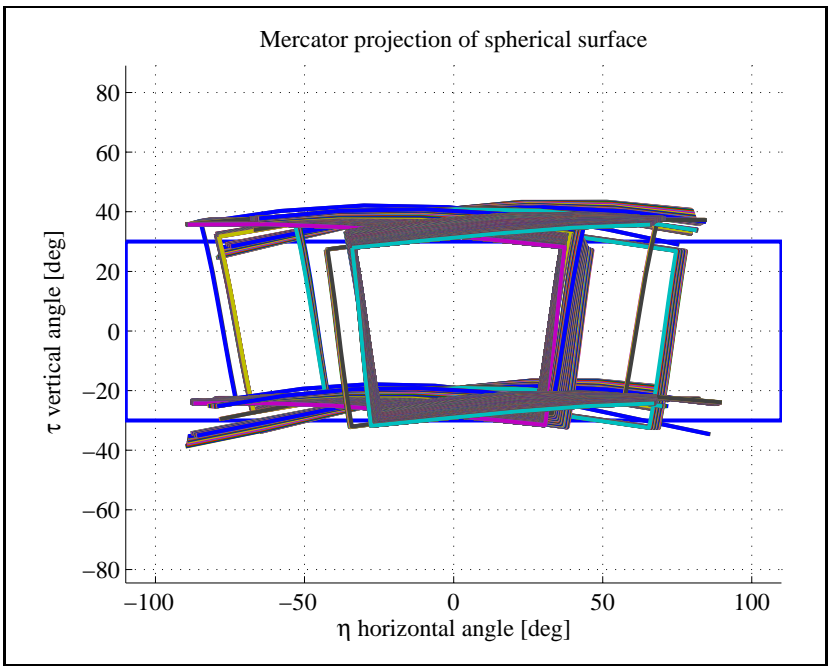

Fig. 8. EFOV with PID C1 $\left(P I D \delta_{a}\right)$ controller

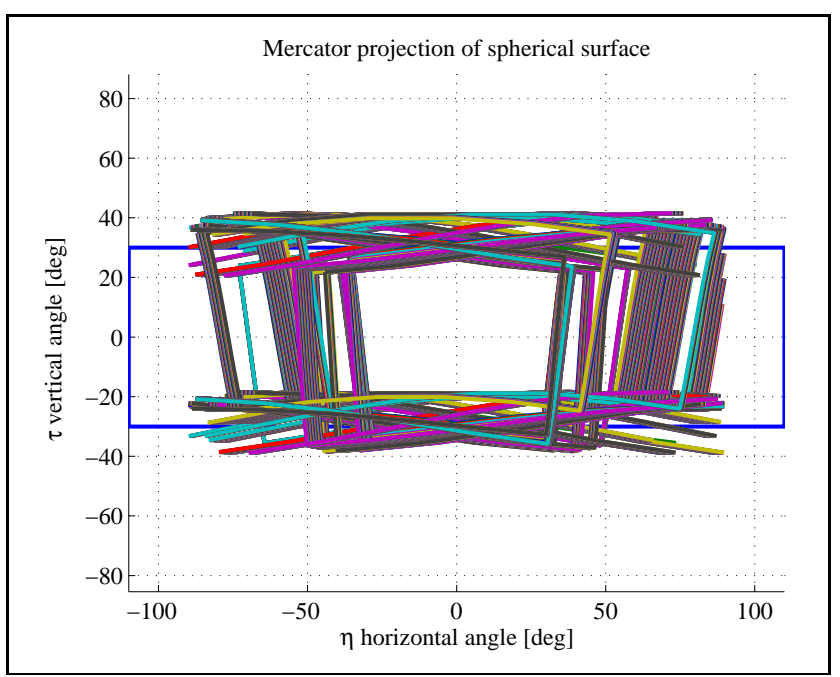

Fig. 9. EFOV with PID C2 $\left(P I D \delta_{r}\right)$ controller

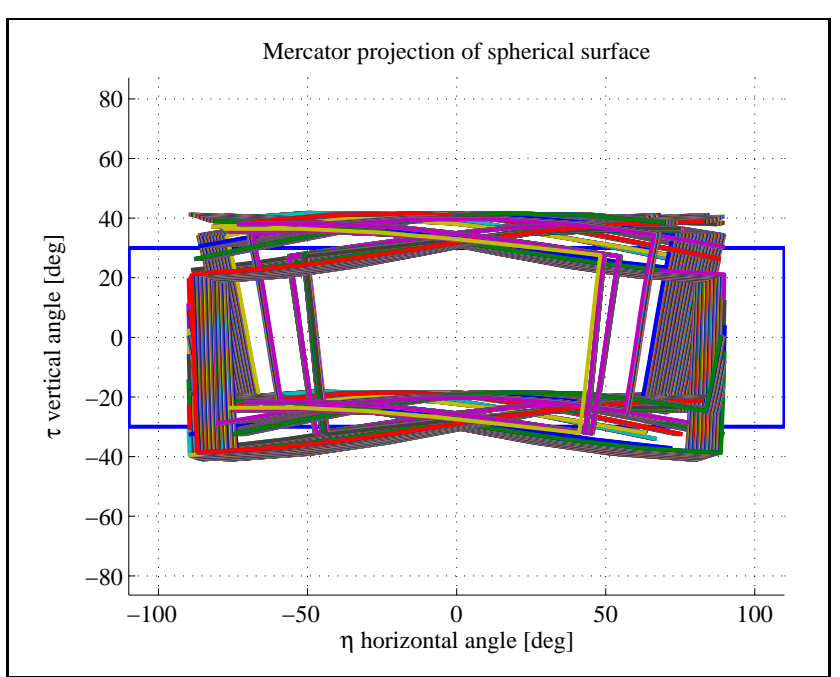

Fig. 10. EFOV with LQ controller

The goal is to track a zig-zag like trajectory with minimum roll angle to provide persistent excitation to the camera based observer and to provide acceptable effective field of view.
The trajectory can be tracked through yaw rate reference tracking meanwhile about zero roll angle should be held. SISO PI(D) controllers and a MIMO LQ controller were tuned to satisfy these requirements. For the SISO controllers two different concept was used: first to control roll dynamics with aileron and yaw with rudder $\left(P I D \delta_{a}\right)$, second to control roll dynamics with rudder and yaw with aileron $\left(P I D \delta_{r}\right)$.

All of the controllers were first tuned on the lateraldirectional linear state space model of the Aerosonde UAV without trajectory tracking. Then they were re-tuned and applied on the nonlinear simulation model of the UAV together with longitudinal control and trajectory tracking. In the nonlinear case all controllers were tuned to provide roll angles between $\pm 10^{\circ}$.

Finally the $P I D \delta_{a}$ solution was the worst with very limited yaw rate tracking capability and so it required a modified trajectory with adverse effect on camera based observer. From the other two methods the LQ one gave the best tracking performance and effective field of view (which was defined in the last section). So as a conclusion it can be stated that the MIMO controller design can better satisfy the requirements for this aircraft.

The future work can be the examination of control solutions with noise and disturbances.

\section{REFERENCES}

[1] B. Vanek, T. Peni, A. Zarandy, J. Bokor, T. Zsedrovits, and T. Roska, "Performance Characteristics of a Complete Vision Only Sense and Avoid System," in in Proceedings of AIAA GNC 2012 (Guidance, Navigation and Control Conference), no. AIAA 2012-4703, Minneapolis, Minnesota, August 2012, pp. 1-15.

[2] Y. Watanabe, "Stochastically Optimized Monocular Vision-based Navigation and Guidance," Ph.D. dissertation, Georgia Institute of Technology, 2008.

[3] L. Matthies, R. Szeliski, and T. Kanade, "Kalman Filter-based Algorithms for Estimating Depth from Image Sequences," Carnegie-Mellon University, Tech. Rep. CMU-CS-87-185, 1987.

[4] S. S. Ponda, R. M. Kolacinski, and E. Frazzoli, "Trajectory Optimization for Target Localization Using Small Unmanned Aerial Vehicles," in In proceedings of AIAA GNC 2009 (Guidance Navigation and Control Conference), no. AIAA 2009-6015. Chicago, Illinois, USA: AIAA, August 2009.

[5] P. Bauer, B. Vanek, T. Peni, T. Zsedrovits, B. Pencz, A. Zarandy, and J. Bokor, "Aircraft Trajectory Tracking with Large Sideslip Angles for Sense and Avoid Intruder State Estimation," in In proceedings of the 22nd Mediterranean Conference on Control \& Automation (MED'14), 2014.

[6] T. Zsedrovits, A. Zarandy, B. Vanek, T. Peni, J. Bokor, and T. Roska, "Collision avoidance for UAV using visual detection," in in Proceedings of IEEE ISCAS 2011 (International Symposium on Circuits and Systems). Rio de Janeiro: IEEE, May 2011, pp. 2173-2176.

[7] R. W. Beard and T. W. McLain, Small Unmanned Aircraft: Theory and Practice. Princeton University Press, 2012.

[8] P. Bauer, Optimal tracking solutions applied to unmanned aerial vehicles. LAP LAMBERT Academic Publishing, 2013.

[9] J. C. Polking. (2000) Mapping the sphere. PDF document. [Online]. Available: http://math.rice.edu/ polking/cartography/cart.pdf 